\title{
Urgences
}

\section{L'esprit du temps}

\section{Luc Bureau}

Numéro 17-18, octobre 1987

L'esprit des lieux

URI : https://id.erudit.org/iderudit/025418ar

DOI : https://doi.org/10.7202/025418ar

Aller au sommaire du numéro

Éditeur(s)

Urgences

ISSN

0226-9554 (imprimé)

1927-3924 (numérique)

Découvrir la revue

Citer ce document

Bureau, L. (1987). L'esprit du temps. Urgences, (17-18), $28-42$.

https://doi.org/10.7202/025418ar

Ce document est protégé par la loi sur le droit d'auteur. L'utilisation des services d'Érudit (y compris la reproduction) est assujettie à sa politique d'utilisation que vous pouvez consulter en ligne.

https://apropos.erudit.org/fr/usagers/politique-dutilisation/
Cet article est diffusé et préservé par Érudit.

Érudit est un consortium interuniversitaire sans but lucratif composé de l’Université de Montréal, l'Université Laval et l'Université du Québec à Montréal. Il a pour mission la promotion et la valorisation de la recherche. https://www.erudit.org/fr/ 


\section{Luc Bureau \\ L'ESPRIT DU TEMPS}

Ce qui fait problème dans la géographie du Canada, c'est I'histoire. Je ne parle pas ici du discours professionnel sur l'histoire, mais de l'histoire réelle. Celle qui enracine et enchaîne les choses, qui les prolonge au-delà des circonstances trop concrètes de leur apparition. Cette histoire qui donne aux événements les plus prosaiques comme les plus élevés un goût d'éternité.

Dans ce dernier sens, autant le dire tout de suite, le Canada est un pays "sans histoire». Les choses y apparaissent sans que l'on puisse en retracer la genèse. Elles s'effacent sans qu'il soit possible d'en percevoir la résonnance. Elles sont contenues toutes entières dans leur immédiateté. On a beau s'y gaver de "Je me souviens" et de "Notre maître, le passé", de "Madeleine de Verchères" et de "Sir Wilfrid Laurier», on sent bien que ces êtres sont sans écho, qu'ils ne sont que formules incantatoires destinées à stimuler des ancrages ou des racines.

Un pays ne vit pas près de quatre cents ans en marge de l'histoire sans que son visage en soit marqué. Ses villes ou ses campagnes, ses palais ou ses gargotes, ses hommes ou ses femmes doivent bien porter l'empreinte de cette absence d'épaisseur. Encore une fois, il ne faut pas être dupe des apparances. Certaines configuratons du paysage, certaines formes institutionnelles, plusieurs vocables servant à la désignaton des lieux peuvent laisser croire à un étirement infini des choses vers des sources éternellement mystérieuses. S'il existe un "mystère canadien", ce n'est pas dans des origines plus ou moins occultées par la viscosité du temps qu'il faut le chercher, mais dans la soudaineté et l'imprévisibilité des événements. Une institution, une oeuvre architecturale, ou tout autre artefact n'acquièrent de valeur qu'à la condition qu'ils s'effacent pour donner la place à quelque chose d'autre. On peut alors en récupérer les débris et leur ménager une niche dans quelque musée de la civilisation, ou en décréter le classement comme monuments historiques. Après les canots d'écorce et les ceintures fléchées, l'école, la religion, la famille et ce qui reste des centres des villes sont d'ores et déjà mûrs pour un tel traitement. Il n'y a place pour le passé que dans les musées. Si la survie d'un pays va de pair avec son degré d'adaptation et sa perte de mémoire, le Canada fait preuve d'une magnifique vitalité.

On doit se permettre ici une digression. Si nous devions ranger le pays dans l'une des trois grandes divisions qui gouvernent la nature 
- les règnes minéral, végétal et animal - nous dirions que le Canada appartient d'emblée au règne végétal. Symboliquement cette contrée est une plante. Mais pas n'importe quelle plante. Seul le champignon lui convient. Dépourvu de racines et de feuilles, éphémère comme un soleil de décembre, le champignon est tout entier dans l'instant de son éruption: sans passé ni futur, il ne se conjugue qu'au présent. Pareilles propriétés nous éclairent sur les réalités canadiennes. Où que la curiosité nous porte, l'épithète champignon nous vient spontanément aux lèvres pour raconter les avatars de ce pays: villeschampignons, banlieues-champignons, Nord-champignon, Ouestchampignon, universités et écoles-champignons, nationalisméchampignon, religion-champignon, partis politiques-champignons, hommes d'affaires-champignons... En supposant que le tout est égal à la somme de ses innombrables parties, le Canada tout entier peut se prévaloir du titre de "pays-champignon".

C'est toujours avec fascination que l'on observe les accomplissements concrets du "champignonnisme" canadien. Tenez, au reżde-chaussée du journal de ce matin, un titre: «Québec ferme Schefferville". La pensée doit suppléer ici aux omissions de cette figure journalistique elliptique. "Québec» n'est qu'un simple raccourci ștylistique pour signifier le gouvernement du Québec, ou encore l'Étàt québécois. "Schefferville» évoque, bien entendu, le nom d'une ville du Nouveau-Québec, une ville comme d'autres villes, avec ses rues, ses magasins, ses cinémas, ses nouveaux-nés, ses chicanes de fámille et ses cordes à linge. Le gouvernement du Québec a donc décidé de "fermer», c'est-à-dire de verrouiller, d'interdire, de proscrire, ou peut-être même de raser la ville de Schefferville. Un titre comme celui-ci risque d'ébranler un esprit obtus, peu habitué aux idées de "génération ou d'anéantissement spontanés". N'en croyant pas ses yeux, ce péquenot ne peut s'empêcher de lire quelques paragraphes du texte d'accompagnement: "La ville de Schefferville est appelée à disparaître avant la fin de l'année, a annoncé en début de soirée hier le ministre des Affaires municipales..., au cours d'une conférence de presse" ( $1^{\text {er }}$ paragraphe); "Le gouvernement fera adopter dans les prochains jours un projet de loi mettant fin à l'existence juridique de Schefferville, a décidé hier le Conseil des ministres" (2 paragraphe). (Le Devoir, 8 mai 1986).

Cette énormité ne laisse pas de nous ébouriffer. Schefferville se voit infliger, en terre canadienne, le remède absolu réservé jadis aux perverses Sodome et Gomorrhe, en terre de légende. Abraham s'approche timidement de la table du Conseil des ministres et fait une dernière tentative: "Est-ce que vous feriez périr le juste avant le méchant? Peut-être y a-t-il cinquante justes dans la ville: les feriezvous périr?" Le Conseil des ministres dit: "Dût-il y avoir trois mille 
justes dans cette ville, que nous la détruirions. Car son péché est immense: les Américains ne veulent plus de son minerai de fer!» (Genèse, versets à peine retouchés par l'auteur).

La brave Maria Chapdeleine voyait dans les "mille noms" familiers donnés aux villages et villes de son pays la preuve éclatante de l'existence de ce dernier. Il lui semblait d'une criante évidence que la dénomination de l'un ou l'autre de ces lieux était toujours liée à une présence réelle. Le village de Honfleur était là, à «huit milles» de leur retranchement en forêt, la Pipe était à "six milles" plus loin, Mistook à «huit autres milles». Puis d'autres noms désignant d'autres lieux tout aussi concrets se tamponnaient dans son esprit... "Trois-Pistoles... Sainte-Rose-du-Dégel... Pointe-aux-Outardes... Saint-André-del'Épouvante... les Escoumains... les Grandes-Bergerones... Gaspé». La conclusion pour Maria était simple: «Dans tout ce pays-ci nous sommes chez-nous... chez-nous!»

Mais qu'arrive-t-il lorsque le nom ne renvoie plus qu'à une absence, quand l'appelé ne répond plus à l'appel... Schefferville... Gagnon... Saint-Paulin-Dalibaire... Saint-Thomas-de-Cherbourg... Saint-Nil... Saint-Octave-de-L'Avenir... Saint-Jean-de-Brébeuf... Saint-Louis-de-Gonzague... Sacré-Coeur-des-Landes... SainteBernadette-de-Pellegrin... Saint-Edmond-de-Pabos... Saint-Gabriel... Saint-Charles-Garnier... Grande-Grève... Grosse-Ile... Rivière-Henry... Saint-Cyriac... Val-Jalbert... Saint-Jean-Vianney... Saint-Ignace...? Interminable litanie de noms qui n'évoquent plus que des fantômes.

S'il est une tentation qui hante l'imaginaire canadien, c'est celle de notre identification au temps plutôt qu'à l'espace. Le temps est notre mesure de toutes choses, aussi bien de l'espérance de vie des objets, des programmes scolaires que des personnes: ils doivent durer tant de temps. Ceci nous amène à de perpétuelles remises en question des configurations existantes, à la recherche de formes nouvelles, au bouleversement des équilibres donnés et à une tension vers de nouveaux équilibres appelés eux-mêmes à se rompre. Notre seule manière de penser le monde est de le penser dans sa succession entrechoquée, dans son existence éphémère. «Mais qu'est-ce que signifie "éphémère»? répéta le petit prince... - Ça signifie "ce qui est menacé de disparition prochaine" (St-Exupéry). Nous avons perdu depuis longtemps la faculté de nous identifier à des lieux durables, à des espaces de références arrêtés. Et s'il est «esprit» que nous vénérions, ce n'est pas celui des «lieux» mais celui du «temps».

\section{L'ESPACE TEMPORALISÉ}

Le temps et l'espace, on le sait, fondent deux des axes majeurs 
de la pensée occidentale. Le temps n'est pas l'histoire, l'espace n'est pas la géographie: ils n'en sont que les supports abstraits. Un peu comme la "nature" constitue pour une profusion d'êtres une trame abstraite de formes, de cataclysmes et de métamorphoses. Pour devenir histoire, le temps exige la collaboration des hommes qui l'arrêtent de s'éparpiller sans ordre et tout de travers ou de s'enrouler sur lui-même comme autour d'une bobine, et lui donnent une direction. Pour devenir géographie, l'espace a également besoin du concours des hommes qui y dessinent leurs clôtures, leurs champs, leurs villes et y élèvent des gratte-ciel et des lapins. De même que l'histoire est l'art de domestiquer le temps, la géographie est l'art d'apprivoiser l'espace. N'empêche que dans le discours quotidien - et à l'échelle de notre planète - temps et histoire, espace et géographie se confondent. lls souffrent de métonymie. Mal dont nous n'essaierons pas ici de les guérir.

Tandis que le temps semble toujours nous être compté, l'espace apparait comme une denrée plus prodigue. Les mots de tous les jours trahissent cet écart perceptuel. Les «je manque de temps», "où trouver le temps?", "ceci prend trop de temps», "le temps file comme une flèche» envahissent les rapports humains. "Perdre son temps" est une faute grave, puisque le temps non utilisé est un "temps mort». "Payer de son temps» est une preuve de générosité exemplaire car «le temps c'est de l'argent». L'horloge, la montrebracelet, le réveille-matin, le calendrier mural, l'agenda sont par exellence les instruments d'orientation de notre société pressurisée. Comme le révèlent plusieurs études, dans les pays développés, plus de $90 \%$ de la population adulte possèdent à la fois une montrebracelet, un réveil et une horloge; et à toutes fins utiles, $100 \%$ de cette même population possèdent une montre. La montre est devenue à ce point partie si essentielle à l'homme moderne qu'il s'arrange pour la porter constamment, y compris dans l'état de nudité intégrale, oubliant même de l'enlever dans ses "travaux" les plus intimes et les plus intemporels comme celui de faire l'amour. Sans risque de se tromper, on peut affirmer que la suppression de la montre ou de ses substituts signifierait l'écroulement immédiat de notre civilisation.

Aucune instrumentation comparable n'est disponible, dans la vie de tous les jours, pour mesurer l'espace. Comme l'écrit Pérec, "(...) on rencontre partout des gens qui ont des montres, et très rarement des gens qui ont des boussoles". Et à moins d'être claustrophobe - ce qui est déjà une maladie!-, on se plaint rarement d'un "manque d'espace». Si l'on en manque, il s'agit le plus souvent d'un espace qualifié: espace salubre, vert, privé... Ce n'est qu'en fonction du temps que l'espace est valorisé. Ainsi, le tracé d'une autoroute en 
plein centre-ville se justifie d'emblée si cette artère permet d'écourter le temps des relations. Les gratte-ciel, les centres commerciaux, les parcs industriels, les carrefours de la finance sont des appareils à comprimer le temps. Les banlieues se soumettent également à la tyrannie de la pendule: les "déplacements pendulaires» ne meublentils pas leur rythme de vie? Toute la morphologie des villes modernes se découpe non pas selon une logique de l'espace mais bien selon une logique du temps. Et les campagnes elles-mêmes adoptent le vocabulaire et les conduites d'une temporalité comprimée, envahies qu'elles sont de «résidences secondaires» - secondaires par rapport a quoi sinon à une durée primaire qui est ailleurs - , d'autobus scolaires chronométrés, de vaches et de petits pois "précoces" et d'agriculteurs à «temps partiel».

Nous assistons ainsi à une formidable dé-géographisation de l'espace, qui nous fait paraître finalement peu audacieux les modèles de «nulle part» élaborés par les grands utopistes de la Renaissance, les More, Campanella et Cie, ainsi que leurs épigones. L'expériencepilote imaginaire que tentait More dans une petite île perdue, au large de quelque côte de l'Amérique latine, nous l'avons réalisée concrètement à une échelle prométhéenne, celle de tout un continent. Les configurations standardisées - dénuées de tout réfèrent symbolique autre que celui d'un fonctionnalisme réducteur - imposées à la quasi totalité des villes et des campagnes de l'Amérique sont l'exemple le plus évident de la disparition de la géographie sur ce continent. $\mathrm{Ce}$ que l'on appelle l'échiquier américain ou canadien n'appartient aucunement à l'esprit des lieux ou de la géographie, mais à celui des mathématiques et de la géométrie qui permettent d'évider l'espace de ses mystères.

\section{DE L'ESPACE ENCHANTÉ A L'ESPACE DÉSENCHANTÉ}

Nous en sommes donc à constater l'évident triomphe, dans nos sociétés, des déterminismes temporels sur les impérialismes géographiques de naguère. Comment une telle substitution s'est-elle accomplie? La réponse à cette question oblige à repérer les principales ruptures de la conscience spatiale au cours des deux ou trois derniers millénaires. Tâche moins lourde qu'on pourrait le croire, puisqu'elle a déjà été réalisée par maints esprits en de maintes occasions. Trois textes de référence suffiront à éclairer les moments fondamentaux de la dérive de la conscience.

Le premier de ces textes - La pensée mythique de Ernst Cassirer - nous renvoie aux commencements, aux prémisses archaiques de l'intuition mythique de l'espace, à ces horizons où d'ailleurs les conceptions de l'espace et du temps ne sont pas dissociées. Une 
même quête de signification illumine la représentation des arrangements spatiaux et de leur ordre temporel.

Si j'interprète bien Cassirer, les choses se passent à peu près ainsi. Le modèle d'intelligibilité et d'action, dans tous les domaines de cet espace-temps mythique, gravite autour de l'idée du Cosmos. Comme l'écrit G. Gusdorf (1967, p. 25), le microcosme humain s'accorde par nécessité au macrocosme sidéral, dans une vision totalitaire de l'univers. L'opposition entre le jour et la nuit, entre la clarté et l'obscurité est ici fondamentale: l'intuition mythique de l'espace se déploie toujours à partir de cette alternance (Cassirer, 1972, p. 123). Elle dicte les configurations, les séparations et les conjonctions des domaines terrestres. Ainsi, le cloisonnement étanche entre le "sacré» et le "profane», donc entre ce qui est éminemment substantiel et redoutable, et ce qui est superficiel et sans conséquence, relève de cette opposition transcendante.

L'est et l'ouest, le nord et le sud ne sont pas de simples repères équivalents servant à s'orienter dans le monde empirique de la perception; «ils possèdent tous au contraire un être spécifique, une signification qui leur est propre, une vie mythique interne». Ce sont des "dieux cardinaux» auxquels, toujours selon Cassirer, la formule de Goethe convient littéralement: «L'Orient est à Dieu, l'Occident est à Dieu. Les terres du Nord et du Sud reposent dans la paix de ses mains" (ld., p.125).

Cette opposition mythique de la lumière et de la nuit se manifeste dans toutes les autres choses terrestres. Ainsi, chez les Romains, c'est en traçant et en croisant des lignes divisant le ciel - appelées dans la langue sacerdotale le decumanus (généralement d'est en ouest) et le cardo (généralement du nord au sud) - que les formes cadastrales et les rapports juridiques régentent la terre des hommes. C'est sur cette même transposition du céleste au terrestre que «repose de développement de la notion de propriété et de la symbolique qui définit et protège la propriété en tant que telle» (Id., p 128). Seule la terre qui est enclose dans des limites stables est sacrée. Il existe d'ailleurs une synonymie entre "sacralisation" et "limitation" de l'espace, dont rend compte linguistiquement l'expression templum (de la racine tem-«couper»): ce qui est découpé, ce qui est délimité. En ce sens, le templum désigne aussi bien le domaine sacré destiné au dieu, que toute parcelle de terre limitée, assignée à un particulier ou à une communauté. "Le tracé de templum a pour conséquence immédiate qu'un esprit prend possession de l'espace ainsi clôturé» (Id., p128). L'espace est toujours ainsi le lieu d'une présence, dont les configurations et les significations font écho à une conscience mythique de l'univers. 
Cette mise en scène de l'espace mythique, où chaque lieu témoigne de la présence d'un esprit, suppose bien sûr une perspective temporelle. Mais cette temporalité est essentiellement régressive, se rapportant au temps fabuleux des "origines», où les choses ont commencé à être ce qu'elles sont et ce qu'elles seront toujours: "Le vrai caractère de l'être mythique ne se découvre que là ou celui-ci apparaît comme être de l'origine. Tout le caractère sacré de l'être mythique se ramène finalement au sacré de l'origine (id., p. 133). L'espace mystique se présente ainsi comme une prolongation créatrice et une justification généalogique de l'installation de l'homme sur la terre.

Insistons enfin sur l'idée que dans tout ce rapport entre la mythologie et l'espace, le terme transcendant est bien le premier: ce n'est pas l'espace qui produit la mythologie mais, à l'inverse, la mythologie qui produit l'espace. Et pour résumer la pensée de Cassirer, nous utiliserons cette phrase de Mircea Eliade: «ll existe pour l'homme une possibilité de transcender le Monde - spatialement, en allant "vers le haut", et temporellement, en allant "à rebours", "à reculons» (1953, p. 27). Élévation et recul. Je vois dans ces deux termes une définition assez réussie du mythe. Je veux de même y voir les conditions essentielles à la diffusion de l'«esprit» dans les lieux.

Deuxième texte: "The Historical Roots of our Ecologic Crisis», de l'historien américain T. Lynn White. Cet article, publié en 1967 dans la superbe revue Science et maintes fois réédité, établit une filiation causale entre notre héritage judéo-chrétien et notre attitude dominatrice et agressive vis-à-vis de la nature. Selon White, la victoire du christianisme sur le paganisme signe la révolution psychique la plus importante de toute l'histoire de la culture occidentale. Cette révolution de l'âme s'accomplit à l'enseigne de deux nouveaux thèmes axiomatiques: 1) l'idée héritée du judaīsme de la domination de l'homme sur la nature: "Soyez féconds et prolifiques (dit Dieu à Adam et Eve), remplissez la terre et dominez-la» (Gen., 1:28); 2) la valorisation d'un nouveau modèle du temps: linéaire, non répétitif, fléché vers un devenir qui contient toutes les promesses du salut. En opposition absolue avec l'ancien paganisme, le christianisme consacre non seulement le dualisme entre l'homme et la nature, mais soutient qu'il y va de l'accomplissement de la volonté de Dieu que l'homme exploite la nature à ses propres fins, jusqu'à ce qu'il parvienne à une parfaite maîtrise de celle-ci.

Dans l'Antiquité mythique et paienne, chaque arbre, chaque filet d'eau, chaque colline avait son propre "genus loci", son esprit tutélaire. Ces esprits, tout en étant accessibles aux hommes, leur 
étaient très différents: les centaures, les faunes, les sirènes se révélaient en effet des êtres bien ambivalents. Avant de couper un arbre, d'endiguer un ruisseau, d'occuper une colline, il était important de se concilier la bienveillance de l'esprit responsable de l'objet ou du lieu. Le christianisme, en répudiant cet animisme paĩen, effaça virtuellement tous les mystères de la nature, et favorisa l'exploitation de cette dernière d'une façon abusive.

Lynn White reconnaît volontiers que son incrimination en bloc de la pensée judéo-chrétienne comme cause première de nos attitudes démystifiantes et agressives à l'égard du monde naturel commande certaines réserves. D'abord, on ne doit pas s'imaginer que l'adoption des valeurs chrétiennes provoqua, au lendemain de la Pentecôte, des bouleversements dramatiques dans les rapports réels entre l'homme et son environnement. C'est par une longue percolation; plusieurs fois centenaire, que la dualité s'engraissa; les paysages de l'homme continuèrent longtemps d'afficher leurs interdits et leurs gnomes. Il y eut aussi, au sein même de l'Église chrétienne, de ces "beatniks" qui se refusèrent à endosser le dogme de la domination de l'homme sur la nature. Le cas le plus merveilleux est assurément François d'Assise qui, toute sa vie durant, essaya de substituer à la monarchie de l'homme sur la création, une sorte de démocratie de toutes les créatures de Dieu: "mon frère le loup", "mon frère le soleil", "ma soeur la fourmi»... On peut dire qu'avec François d'Assise les lieux et les créatures du monde retrouvent leur âme. Mais ce fut une victoire aux effets très limités dans le temps et dans l'espace. Car la vision de saint François était tellement hérétique qu'un général de l'Ordre des Franciscains, saint Bonaventure, essaya de supprimer par la suite les premières relations, un peu trop hippies à son goût, du célèbre "Petit pauvre".

Les modalités actuelles de la relation de l'homme à ses «lieux» ne sont donc pas le résultat de l'irruption d'une pensée récente, à peine dégagée des cendres chaudes de la révolution industrielle. Nous courtisons au contraire une idée qui a deux mille ans d'âge. Côté pile, cette idée dé-paganise le lieux et les objets du monde et en fait des abstractions aseptisées. Côté face, elle propose à l'homme d'habiter le temps plutôt que l'espace, de voir les choses dans leur devenir plutôt que dans leur permanence. Il convient cependant de relativiser dans le temps l'incidence de cette idée sur l'espace: jusqu'aux tournants des XVIIle et XIX ${ }^{e}$ siècles, les hommes n'avaient sans doute pas les moyens de leur idée. Il semble aujourd'hui que l'idée ait été largement débordée par les moyens. Drôle d'ironie: c'est en nous déchristianisant spirituellement que nous adoptons avec le plus de ferveur le dogme chrétien de la suprématie de l'homme sur son environnement. 
Troisième texte: The Reenchantment of the World, de Morris Berman, professeur d'histoire à l'Université de Victoria (B.C.). Je me permettrai, encore une fois, de ne mobiliser que les articulations les plus névralgiques de cet ouvrage de 366 pages.

La grande fracture historique, le moment sacrilège où l'Occident se met en marche vers son destin prométhéen, s'accomplit, selon l'évaluation de Berman, sous les lampadaires de la révolution scientifique des $X V l^{e}$ et XVII siècles. La vision du monde qui prédominait jusqu'à la veille de cette révolution était celle d'un monde enchanté: les rochers, les arbres, les rivières et les nuages étaient tous perçus comme merveilleux, animés, et l'homme se sentait chez-lui dans cet environnement. On n'y observait aucune distinction. Le cosmos, en un mot, appartenait à l'homme et l'homme au cosmos: ce que Berman appelle une "conscience participante» au monde.

Puis il y eut Bacon et son Novum Organum (1620), Galilée et sa condamnation par le tribunal de l'Inquisition (1633), René Descartes et son Discours de la Méthode (1637), Isaac Newton et ses Principia (1687). Bacon montre qu'on doit questionner la nature directement, en la plaçant dans une situation telle qu'elle est obligée de formuler une réponse: Natura Vexata, lance-t-il, "nature vexée» ou «nature tourmentée», contrainte à dire "oui» ou «non" et à perdre ses mystères. Galilée, dans un texte célèbre, annonce lui aussi le nouveau paradigme de la domination de la nature:

La philosophie est écrite dans ce vaste livre constamment ouvert devant nos yeux (je veux dire l'univers) et on ne peut le comprendre si d'abord on n'apprend à connaître la langue et les caractères dans lesquels il est écrit. Or il est écrit en langue mathématique, et ses caractères sont le triangle et le cercle et autres figures géométriques, sans lesquelles il est humainement impossible d'en comprendre un mot... (Oeuvres t. Vl, p. 232). Le philosophe Cogito réduit quant à lui tous les phénomènes du monde sensible à deux qualités fondamentales: l'étendue et le mouvement; tout le reste (dureté ou douceur, couleur, odeur, goût) n'est pour Descartes que broutille, mystification. Newton nous renvoie pour sa part aux pommes désenchantées, qui n'ont plus d'autres vertus que de se détacher de l'arbre et de tomber au sol; la Création tout entière est soumise à une seule loi: celle de la pesanteur ou de la gravitation universelle.

À travers l'itinéraire philosophique et scientifique des $X V l^{\mathrm{e}}$ et $X V I l^{e}$ siècles s'enclenche ainsi le retrait progressif de l'esprit de tous les phénomènes sensibles. La connaissance de l'univers devient 
synonyme de raison, d'expérimentation, de mesure précise. Le monde n'est plus à la contemplation mais à l'action, à sa transformation par la technique. C'est cette situation d'une philosophie devenue une technologie qu'anticipe, vers 1590, Christophe Marlowe dans son récit populaire La Tragique Histoire du Docteur Faustus. Faust cherche à se rendre maître de l'univers: «Apprends de moi maintenant, lui susurre Méphisto, à faire le tonnerre, l'éclair, la grêle, la neige et la pluie, à déchirer les nuages, à secouer la terre et les rochers escarpés et à les faire éclater en deux, à enfler les mers, à les faire rugir et sortir de leur lit... Apprends, Faust, à voler comme moi-même, aussi rapide que la pensée, d'un royaume à l'autre...» Bacon, Galilée, Descartes ou Newton ont plus de points en commun avec Faust qu'avec François d'Assise. Ne sommes-nous pas les héritiers légitimes de ces "hommes faustiens"?

Deux ruptures: l'avènement du christianisme et la révolution scientifique des $\mathrm{XV}^{\mathrm{e}}$ et $\mathrm{XVIl}{ }^{\mathrm{e}}$ siècles. Trois manières non concordantes pour la conscience de se donner le monde: l'expérience mythique où les lieux et la conscience se confondent, la vision chrétienne où les lieux ne sont que des symboles qui renvoient à la transcendance divine, la vision faustienne où les lieux ne composent plus qu'un support idoine à l'agir de l'homme.

$\grave{A}$ chacun de ces moments de la conscience spatiale correspond une vision appropriée du temps: temps enroulé sur lui-même des origines mythiques, temps linéaire et eschatologique de la chrétienté, temps faustien de l'éternel progrès de l'ère moderne, dont Perrault - celui des Contes et de la querelle des Anciens et des Modernes - proclame au XVIle siècle la victoire. "Le genre humain, (certifie Perrault), doit être considéré comme un seul homme éternel, en sorte que la vie de l'humanité, comme la vie de l'homme, a eu son enfance et sa jeunesse, qu'elle a actuellment sa virilité, mais qu'elle n'aura pas de déclin" (Parallèles des Anciens et des Modernes). Fi, l'Apocalypse!

Tout ceci constitue pour moi une sorte de canevas ou de grille de référence, avec en "ordonnée» les figures successives du temps, et en «abscisse» les figures concomitantes des lieux. II ne faudrait pas croire cependant que chacune de ces figures annule celles qui l'on précédée: la linéarité de l'éternel progrès n'annule pas l'image d'un temps qui tourne en rond, tout comme le «lieu/support» n'anéantit pas le lieu mythique. Ce que nous avons voulu faire jusqu'ici, c'est d'insister sur les tendances lourdes, et en particulier sur celle qui prédomine en Occident depuis au moins trois ou quatre siècles. Mais même en cette époque de profonde désacralisation du monde, il survient ça et là des expériences où les lieux signalent leur présence et 
exercent leur pouvoir de transfiguration. Nous en voyons de précieux exemples dans la littérature contemporaine.

\section{POUVOIR DE MÉDIATION DE LA VLLE}

Il peut sembler, aujourd'hui, incohérent de parler du pouvoir de médiation, d'inspiration et même de transfiguration de la ville. Autoroutes. Parkings. Béton. Fast-foods. Tintamarre démentiel. On ne peut trouver cadre plus alinéant et débilitant. Mais les images de la ville ne se réduisent pas seulement à ses décors extérieurs.

Il arrive parfois, il arrive souvent que la ville se laisse reconnaître par une conscience qui la pénètre dans ses replis les plus intimes. Comme en une sorte de symbiose, la conscience s'immerge dans la ville et cette dernière s'imbibe par capillarité dans la conscience. Les résultats de cette «transfiguration» réciproque ne sont jamais nuls: la conscience en ressort à tout jamais marquée, et par delà la ville elle-même. La ville et la conscience se découvrent l'une par l'autre.

Que de mystiques, poètes et rêveurs ont connu une semblable expérience de la ville: saint Augustin, Montaigne, Hugo, Balzac, Zola, Rimbaud et Baudelaire disant de Paris: «Tu m'as donné ta boue et j'en ai fait de l'or". Je retiens ici, afin d'illustrer mon propos, un petit essai sur la ville d'un très grand écrivain contemporain: La forme d'une ville de Julien Gracq. Publié en 1985, ce texte m'apparaît comme une oeuvre entièrement de facture géographique, mais d'une géographie subtile et inspirée, rarement égalée.

À partir de la lecture de Gracq, je conçois divers modes de représentation de la ville, que j'associe aux figures symboliques du héros, de l'eunuque et du poète. Je n'insisterai pas sur les deux premières de ces figures, sauf pour les démarquer par rapport à la troisième.

Il prend deux choses pour que naissent des héros de la ville: un grand cloaque où croupissent les hommes, et des êtres capables de s'élever au-dessus de ce bourbier et de le dominer. Or à qui veut la voir ainsi la ville présente tous les attributs essentiels de la confusion chaotique. Surgissent alors des êtres qui, répondant à leur propre appel, décident de dompter ces lieux de malheur, de les nettoyer, de les bonifier, bref de les aménager et de les maitriser. La ville devient pour eux un vaste champ de bataille où s'exerce leur art. Leur rapport à celle-ci est à sens unique: ils la dominent, elle se soumet. Le drame de la ville est tout extérieur à eux-mêmes.

Si la ville est vécue par le héros, non pas en symbiose, mais sous 
le signe d'une domination unilatérale, l'eunuque de la ville quant à lui est doublement inerte: il ne peut ni agir sur elle ni agir par elle. L'esprit de l'eunuque est en effet impuissant à féconder la ville, et celle-ci n'éveille dans sa conscience aucun appétit particulier. Esprit aliéné et ville aliénante. On trouve dans la vie quotidienne ainsi que dans la littérature moderne de multiples traces de cette dépossession, où l'on se "victimise» à qui mieux mieux.

Reste la ville du poète, du rêveur, de l'enfant ou du mythomane. C'est de cette ville, plus imaginée que concrètement dominée, plus secrète que connue et rationalisée, dont il est question dans le livre de Gracq. L'auteur, comme il le dit lui-même, n'entend pas tracer le portrait d'une ville - en l'occurence Nantes - où il vécut, en réclusion quasi totale dans un lycée, sept années de sa vie d'enfance et d'adolescence. Non, la ville de Gracq n'est ni celle des proliférations monumentales et architecturales, ni celle des configurations paralysées des plans d'urbanisme, ni celle des échanges lucratifs, ni celle des souvenirs mélancoliques qu'on transporte avec soi comme des chaînes, mais plutôt celle des étranges combinaisons qui se nouent entre deux coeurs: le sien et celui de la ville. Dès le premier paragraphe du livre, le pont symbiotique est jeté: "La forme d'une ville, écrit-il, change plus vite... que le coeur d'un mortel. Mais... il arrive aussi, il arrive plus d'une fois que ce coeur, elle l'ait changé à sa manière, rien qu'en le soumettant tout neuf encore à son climat et à son paysage, en imposant à ses perspectives intimes comme à ses songeries le canevas de ses rues, de ses boulevards et de ses parcs" (p.1). Quelques pages plus loin, en précisant les objectifs de son essai, Gracq insiste à nouveau sur l'indicible rapport: "Je voudrais seulement essayer de montrer... comment elle (la ville) m'a formé... et comment de mon côté... je l'ai remodelée selon le contour de mes rêveries intimes, je lui ai prêté chair et vie selon la loi du désir plutôt que selon celle de l'objectivité» (p.7).

Comment s'opère donc cette commune union entre la ville et l'esprit? Je ne compte aucunement épuiser ici l'oeuvre de Gracq par une sorte de recension exhaustive des manoeuvres de l'esprit et des incitations du paysage urbain. Je me contenterai de fournir certains exemples de cette prodigieuse alchimie en assemblant et en tirant quelques passages significatifs.

\section{La ville comme schéma initiatique}

C'est sans doute l'un des attributs potentiels les plus essentiels de la ville d'être un «milieu incubateur» où l'enfant apprend à «auner» le monde: 
J'entrais en sixième: j'avais onze ans. À demi-connue, à demi-rêvée, la ville ne s'est jamais dégagée de ce pli imprimé dès mon premier contact avec elle. (p.4)

Il se trouve d'ailleurs que le cours des choses, dans le dernier demisiècle, me protège contre tout démenti infligé par la réalité à l'image empreinte en moi de cette ville, qui a été le milieu incubateur de mon adolescence. (p.8)

Au moment même où s'achevait et se fermait, à dix-huit ans, un des cycles de ma vie, la ville scellait ainsi elle-même par son avatar cet ensemble clos du souvenir. (p.9)

La chance a fait de ces années de mon enfance et de mon adolescence un gisement que la vie a monnayé, une richesse toujours mobilisable que je prodigue à mon gré sans m'en sentir jamais plus pauvre. (p.10)

J'essaie de prendre quelque distance avec ce complexe de rues et de places dans lequel ma vie s'est trouvée coulée à son époque la plus sensible: entreprise aléatoire, car ce qui a touché de près à nos commencements ne cesse jamais tout à fait, même dans l'absence, de participer de loin, si peu que ce soit, à nos mutations. (p.199)

Il y a dans ces quelques extraits une étonnante leçon de géographie urbaine. La ville que nous côtoyons tous les jours n'est jamais celle qui est déposée devant nous avec ses formes et ses objets matériels. Cette ville-là n'est qu'un mirage, ou tout au plus une pâte à modeler, une prolifération de possibles. La vraie ville, celle qui nous accompagne partout où nous allons, que nous projetons sur tous les lieux que nous fréquentons, est celle sans cesse réactivée de notre patrimoine imaginaire, hérité des rêveries de l'enfance et de l'adolescence.

\section{La ville comme réservoir de secrets}

L'ambition de la science positive est d'en arriver à franchir l'opacité des choses, à faire en sorte qu'elles deviennent transparentes comme du cristal: plus de secrets, plus de mystères, la sainte nudité adamique. Ce n'est pas ainsi que Gracq voit la ville: la fascination qu'elle exerce sur lui est proportionnelle aux intimités qu'elle lui refuse.

Il n'est pas nécessaire, il est sans doute même de médiocre conséquence qu'on l'ait (la ville) vraiment habitée. Plus fortement, plus durablement peut-être, agira-t-elle sur nous si elle s'est gardée en partie secrète, si on a vécu avec elle... sans accès vrai à son intimité familière... Pour s'être prêtée sans commodité, pour ne s'être jamais tout à fait donnée, peut-être a-t-elle enroulé plus serré autour d'elle, comme une femme, le fil de notre rêverie, mieux jalonné à ses couleurs les cheminements du désir. (p.1-2)

Je vivais au coeur d'une ville presque davantage imaginée que connue, 
où je possédais quelques repères solides, où certains itinéraires m'étaient familiers, mais dont la substance, l'odeur même, gardait quelque chose d'exotique: une ville où toutes les perspectives donnaient d'ellesmêmes sur des lointains mal définis, non exploités, un canevas sans rigidité, perméable plus qu'un autre à la fiction. (p.4)

Une ville qui vous reste aussi longtemps à demi interdite finit pas symboliser l'espace même de la liberté. (p.5).

Cette vie qui passait au large, qui me frôlait sans cesse de son courant, et pourtant me laissait échoué sur la grève, animait pour moi jusqu’à l'obsession les rues d'une cité dont je ne percevais que la rumeur. (p.6)

Attention! semble dire Gracq, vous, urbanistes, géographes, cartographes, sociologues, ingénieurs, qui entendez livrer aux hommes tous les mystères de la ville, vous ne faites que la violer, la dévaliser, la banaliser. Une ville sans secret est comme une stripteaseuse qui se présente nue sur scène: il ne lui reste plus qu'à se ré-habiller.

\section{L'intemporalité du produit de l'imaginaire urbain}

L'image mentale qui jaillit dans notre esprit, à l'évocation du nom d'une ville, ne connaît pas les lois de la linéarité temporelle, avec des strates superposées des ères géo-psychologiques antérieures. Gracq rend compte de cette confusion des temps.

L'ancienne ville - l'ancienne vie - et la nouvelle se superposent dans mon esprit plutôt qu'elles ne se succèdent dans le temps: il s'établit de l'une à l'autre une circulation intemporelle qui libère le souvenir de toute mélancolie et de toute pesanteur; le sentiment d'une référence décrochée de la durée projette vers l'avant et amalgame au présent les images du passé au lieu de tirer l'esprit en arrière. (p.9).

Mais, pendant que j'écris ces lignes teintées de regret [Gracq évoque ici la disparition depuis plus d'un quart de siècle des transways dans les rues de Nantes], un journal local publie une nouvelle surprenante: les tramways de Nantes vont revenir! [...] Nouvelle propre à me confirmer dans l'idée, qui ne me quitte pas au long de ces pages, d'un temps réversible, d'un pouvoir de résurrection propre à ce passé de Nantes... (p.21).

La cité est peuplée, encore aujourd'hui, pour moi, non de lieux célèbres, mais d'endroits où j'aime me tenir, parfois (tant le présent et le passé se mêlent confusément dans le sentiment que j'ai de Nantes) matériellement, parfois en souvenir. (p.111).

L'empreinte gravée dans l'esprit de la ville n'a pas besoin d'être rappelée, comme un souvenir qu'on exhumerait péniblement de la mémoire; elle est toujours en état de mobilisation.

La géographie de la ville, comme en témoigne superbement 
Gracq, est donc avant tout donnée dans les rêveries auxquelles elle s'accommode. Bien d'autres exemples, toujours empruntés à Gracq, pourraient servir à illustrer une géographie onirique de la ville, où les chapitres s'intituleraient: L'essence d'une ville n'est pas dans ses mouvements. La ville n'existe que pour Moi. Interférence obligée de la poésie et de la géographie. Le sortilège des noms de la ville, et quoi encore!

\section{CONCLUSION}

Dire que les lieux n'ont plus d'esprit - pris qu'ils sont par «le vertige de métamorphose" qui est à la marque de notre siècle - ce serait s'absoudre un peu vite de nos fautes à l'égard de l'imaginaire, de la "phantasia", du rêve, que nous avons réduits à des productions inférieures sinon dégradantes de l'esprit. En fait, ce sont moins les lieux qui ont perdu l'esprit, que nous qui avons séquestré le nôtre. Ni les prouesses scientifiques et technologiques, ni les incantations et neuvaines écologiques, ni les législations et règlementations étatiques ne peuvent prévenir ou guérir la défiguration des lieux de l'homme. Ici, plus qu'ailleurs, la célèbre parole empruntée par Heidegger au poème de Hölderlin prend tout son sens: [...] l'homme habite en poète..." Il est plus difficile de se conformer à cette prescription du philosophe et du poète que de fabriquer des bébéséprouvettes ou de vaincre les pluie acides.

OUVRAGES DE RÉFÉRENCE

Berman Morris: [1984] The Reenchantment of the World, New-York, Bantam Books. 366 p.

Cassirer Ernst: [1972] La philosophie des formes symboliques. 2. La pensée mythique, Paris, Les Éditions de Minuit, $342 \mathrm{p}$.

Éliade Mircea: [1978] Mythes, rêves et mystères, Paris, Gallimard, 279 p.

Gracq Julien: [1985] La forme d'une ville, Paris, José Corti, 213 p.

Gusdorf Georges: [1967] Les sciences humaines et la pensée occidentale. Il. Les origines des sciences humaines, Paris, Payot, $500 \mathrm{p}$.

Heidegger Martin: [1980] Essais et conférences, Paris, Gallimard, 349 p.

White Lynn: [1967] «The Historical Roots of our Ecologic Crisis», Sclence 155, 1203-1207 p. 